\title{
Protocol and baseline data for a prospective open-label explorative randomized single- center comparative study to determine the effects of various intravenous iron preparations on markers of oxidative stress and kidney injury in chronic kidney disease (IRON-CKD)
}

Ahmed Ziedan and Sunil Bhandari (i)

\begin{abstract}
Background: Intravenous (IV) iron is frequently used to treat iron deficiency/anemia in patients who are unable to tolerate oral iron or the oral iron is not sufficient toreplete iron requirements. However, safety concerns regarding the potential increase in oxidative stress and other adverse effects persist and it remains unclear whether all iron preparations are equivalent. Indeed, the comparative risk of adverse events with IV iron preparations has not been extensively assessed. We hypothesize that IV iron leads to changes in oxidative stress, endothelial function, and potential renal damage depending on the iron formulation (related to the generation of "free" or catalytic labile iron) and this may result in more tubular and glomerular injury manifested as increased proteinuria and raised neutrophil gelatinase-associated lipocalin (NGAL) levels in patients with chronic kidney disease (CKD).

Methods: IRON-CKD is a prospective, open-label, explorative, randomized, single-center study designed to compare the safety and efficacy of three parenteral iron preparations: low-molecular-weight iron dextran-Cosmofer, iron sucroseVenofer, and iron isomaltoside-Monofer. The study includes 40 adults who have established CKD stages 3-5 and serum ferritin (SF) of less than $200 \mu \mathrm{g} / \mathrm{L}$ or transferrin saturation (TS) of less than 20\% (or both); they were randomly assigned in a 1:1:1:1 ratio to $200 \mathrm{mg}$ iron dextran, $200 \mathrm{mg}$ iron sucrose, $200 \mathrm{mg}$ iron isomaltoside, or $1000 \mathrm{mg}$ iron isomaltoside. After randomization, participants undergo baseline assessments and then an iron infusion. Each participant is followed up at $2 \mathrm{~h}$, day 1 , week 1 , and months 1 and 3 . At each follow-up visit, patients undergo clinical review, measurement of pulse wave velocity (PW), blood tests for renal function, and collection of serum/plasma samples for oxidative stress and inflammatory markers.

The primary outcomes are measures of oxidative stress, inflammatory markers, and markers of acute renal injury in comparison with baseline measures of each iron preparation and between each of the iron preparations. Secondary objectives include effects on hematinic profiles and hemoglobin concentrations, changes in arterial stiffness, incidence of significant side effects, and change in patients' quality of life.

(Continued on next page)
\end{abstract}

* Correspondence: sunil.bhandari@hey.nhs.uk

Hull University Teaching Hospitals NHS Trust and Hull York Medical School,

Hull Royal Infirmary, Anlaby Road, Hull HU3 2JZ, UK

(C) The Author(s). 2019 Open Access This article is distributed under the terms of the Creative Commons Attribution 4.0 International License (http://creativecommons.org/licenses/by/4.0/), which permits unrestricted use, distribution, and reproduction in any medium, provided you give appropriate credit to the original author(s) and the source, provide a link to the Creative Commons license, and indicate if changes were made. The Creative Commons Public Domain Dedication waiver (http://creativecommons.org/publicdomain/zero/1.0/) applies to the data made available in this article, unless otherwise stated. 


\begin{abstract}
(Continued from previous page)
Results: Between October 2015 and April 2018, 521 individuals were identified as potential participants; 216 were contacted, 56 expressed an interest, 49 attended a screening visit, and 40 were confirmed to meet the eligibility criteria and were randomly assigned. The mean age was 58.3 (standard error of the mean 4.4) years, and 23 (58\%) were male. All patients were white and English-speaking. The mean SF was $66.6 \mu \mathrm{g} / \mathrm{L}$, TS was 21.2\%, and hemoglobin was $121.6 \mathrm{~g} / \mathrm{L}$ at randomization for the whole group. The mean estimated glomerular filtration rate was $27.8 \mathrm{~mL} / \mathrm{min}$, the urinary protein/ creatinine ratio was $104.3 \mathrm{mg} / \mathrm{mmol}$, and CRP was $6.65 \mathrm{mg} / \mathrm{L}$.

Discussion: IRON-CKD will provide important information on the short-term effects of three preparations of IV iron in CKD patients with biochemical functional or absolute iron deficiency on measures of oxidative stress, inflammation, endothelial function, and renal injury.
\end{abstract}

Trial registration: European Clinical Trials Database (EudraCT) number 2010-020452-64.

Keywords: Acute kidney injury, Chronic kidney disease, Cosmofer, Intravenous iron, Iron deficiency, Monofer, Oxidative stress, Protocol, Randomized trial, Venofer

\section{Background}

Chronic kidney disease (CKD) is a worldwide public health problem that affects about $4-6 \%$ of the UK adult population and is associated with a high prevalence of cardiovascular disease and high economic cost [1]. Patients have either absolute (depletion of both circulating and iron stores) or functional (depletion of circulating/available iron) iron deficiency. Currently, intravenous (IV) iron is used regularly in patients with CKD to correct anemia and optimize the use of erythropoietin-stimulating agents $[2,3]$.

Debate remains regarding the effects of IV iron on oxidative stress, renal function, and proteinuria in patients with CKD. Clinical data on the effects on renal function are encouraging [4]. However, assessment of transient damage and markers of potential damage are required to confirm these clinical findings.

Critical to the importance of iron in biological processes is its ability to cycle reversibly between its ferrous and ferric oxidation states. This precise property, which is essential for its functions, also makes it dangerous because "free" (labile) iron can catalyze the formation of free radicals leading to cell damage. Labile iron (catalytic iron) consists of chemical forms that can participate in redox cycling. This property makes iron potentially hazardous by enabling it to participate in the generation of powerful oxidant species (such as hydroxyl radical) (metal-catalyzed Haber-Weiss reaction) or reactive iron-oxygen complexes (such as ferryl or perferryl ion) or both [5]. Iron also has a major role in the initiation and propagation of lipid peroxidation by either catalyzing the conversion of primary oxygen radicals to hydroxyl radicals or forming a perferryl ion. This pool of labile iron is increased in many disease states. Certain iron chelators may provide a protective effect, thus establishing a cause-effect relationship, at least in animal models [6]. Several animal models have demonstrated the potential detrimental effects of "free" iron on glomerular function [7]. However, there are limited human data. Shah et al. compared the effects of catalytic iron in subjects with either no renal disease or diabetes with patients with diabetes and managed to demonstrate that patients with overt diabetes have an increase in urinary catalytic iron [6]. In their preliminary studies, the authors showed that treatment with the chelator deferiprone leads to a reduction in proteinuria in patients with diabetic nephropathy and glomerular diseases which is unresponsive to other treatments [6, 7]. Lin et al. have also shown that chelation therapy with ethylenediaminetetraacetic acid (EDTA) in patients with CKD results in a reduced rate of decline in the glomerular filtration rate [8]. The authors attributed the beneficial effect to the chelation of lead, which also participates in the Fenton reaction. However, given the affinity constants for iron and lead, the large experimental evidence for the role of iron in kidney disease and the demonstrated efficacy of EDTA in enhancing excretion of urinary iron suggest that the beneficial effects are more likely to be explained by the chelation of iron rather than lead [8].

When IV iron is administered, it passes to the reticulo-endothelial system (RES). The iron complex with dextran, isomaltoside, or sucrose splits. Iron is therefore combined to ferritin or transferrin, which is used in hemoglobin $(\mathrm{Hb})$ production and storage. Iron dextran consists of ferric oxyhydroxide and polymerized dextran; the former passes to the RES and is eliminated from the plasma at $10-20 \mathrm{mg} / \mathrm{h}$ as it is released from the dextran complex to bind to transferrin and pass to the erythroid bone marrow [9]. Iron sucrose consists of soluble iron, hydroxide, and sucrose, which pass to the RES and then to the bone marrow. However, a small percentage of iron may be released as free iron. This may be toxic to cells, in particular glomerular and mesangial cells via oxidative stress (lipid peroxidation) and cell cytotoxicity, leading to endothelial dysfunction, which leads to proteinuria, accelerated atherosclerosis, and 
potentially increase in serum creatinine. This oxidative stress also leads to a reduction in ATP production via mitochondrial damage. Previously, in animal studies, Zager et al. have demonstrated that sucrose-based iron preparations may cause direct cytopathic changes to renal cells and this therefore potentially causes renal deterioration [10-12].

Iron isomaltoside consists of a linear and unbranched oligosaccharide carbohydrate moiety where the iron is tightly bound in a matrix structure. This enables a controlled and slow release of iron to iron-binding proteins and passage to the RES, thus avoiding potential toxicity from the release of labile iron. The strongly bound iron within the iron isomaltoside formulation allows flexible dosing over a short time period. Compared with compounds in which iron is more loosely bound in the complex, the iron isomaltoside complex potentially leads to the generation of less oxidative stress and less immunological toxicity [13].

Neutrophil gelatinase-associated lipocalin (NGAL) is a $25-\mathrm{kDa}$ glycoprotein that is normally found in neutrophils, hepatocytes, and proximal tubular cells [14, 15]. Its expression is increased in conditions such as inflammation and infection. High levels of secretion into the blood and urine upon injury to the kidney are seen, for example, during renal ischemia, which occurs after cardiopulmonary bypass and in critically ill patients [16]. It has been shown that urinary NGAL and plasma NGAL are diagnostic of acute kidney injury (AKI) in critical illness with a sensitivity and specificity of more than $85 \%$ [14-18]. Therefore, NGAL may be a useful early marker of AKI. NGAL levels are increased within hours of injury. This therefore could help elucidate whether IV iron therapy potentially causes acute renal injury, which goes undetected and gives further insight into the etiology of renal injury. NGAL therefore may be a good indication of renal injury but not necessarily of renal function. Of course, NGAL is increased in the blood and urine in other conditions which may serve as confounders, such as inflammation and infection and various types of cancer. However, specific levels have been determined for these. Furthermore, the combined use of other oxidative stress and inflammatory markers will help advance the field of biomarkers of drug kidney toxicity.

\section{Research question}

The IRON-CKD trial has been designed to assess the effects of three preparations of IV iron (Cosmofer, Venofer, and Monofer) in a cohort of CKD patients with biochemical functional or absolute iron deficiency on changes in oxidative stress, inflammation, and potentially acute effects on renal function. In addition, the differential effects of low-dose and high-dose iron isomaltoside (Monofer) on the various parameters will be assessed.

\section{Primary, secondary, and other objectives}

As this is an "explorative" study, the primary objective is to provide valuable information on the following outcomes of interest:

1. oxidative stress and inflammatory markers

2. measures of acute renal injury, including serum creatinine, estimated glomerular filtration rate (eGFR), urinary proteinuria, and NGAL

3. measures of iron status (hematinic profiles) and response to treatment

4. $\mathrm{Hb}$ response to IV iron

5. effects of IV iron on endothelial function assessed by pulse wave velocity (PWV)

6. change in patients' quality of life

7. documented acute clinical side effects as a result of iron infusion.

\section{Primary outcome measures}

1. measures of oxidative stress

2. inflammatory markers

3. markers of acute renal injury in comparison with baseline measures and between iron preparations.

\section{Secondary outcome measures}

1. effects on hematinic profiles

2. effects on $\mathrm{Hb}$ concentrations

3. changes in arterial stiffness

4. incidence of significant side effects

5. change in patients' quality of life.

\section{Study design}

This is an investigator-led, open-label, single-center, prospective, randomized explorative study involving patients attending nephrology outpatient clinics for a screening visit (visit 1) and five study follow-up visits, including the baseline visit.

\section{Ethics}

This study was carried out in accordance with good clinical practice guidelines and the Declaration of Helsinki and received ethical approval from the Northern Regional Ethics Service (NRES) Committee Yorkshire and the Humber - Leeds East, UK (approval reference number $10 / \mathrm{H} 1306 / 40)$. If participants should suffer harm due to the study, the trial is covered by the National Health Service (NHS).

\section{Indemnity insurance}

The majority of patients attending the service are white British as $92 \%$ of the geography of the region is white. Therefore, no non-white participants were recruited in 
view of the potential confounder with inflammatory measures and increased cost associated with translated information leaflets.

Iron replacement is initiated on the basis of a set protocol which is in accordance with Renal Association and NICE (National Institute for Health and Care Excellence) guidelines [19]. Potential participants attending the nephrology clinic in Hull Royal Infirmary were invited to take part in the study. Written information was provided and signed consent was obtained from each patient. Serum ferritin (SF), iron, and transferrin saturation have been monitored at baseline and throughout the study in accordance with protocol.

Conventional additional therapies were adjusted as deemed necessary for best clinical practice. Oral iron was stopped for the duration of the study. Participants were randomly allocated to receive one of four iron therapies and will be followed up at $2 \mathrm{~h}$, day 1 , week 1 , and months 1 and 3 (Fig. 1).

\section{Eligibility}

We recruited 40 adults participants with CKD (stages 3-5) and functional or absolute iron deficiency (SF level $<200$ $\mu \mathrm{g} / \mathrm{L}$ or transferrin saturation (TS) $<20 \%$ or both) who were due to receive therapeutic IV iron on the basis of the inclusion and exclusion criteria and who had not had an iron infusion in the last 6 weeks (Table 1). The exclusion criteria were designed to identify participants for whom the safety of iron infusion may have been a concern or those with potential confounding factors to NGAL or iron marker measurements such as cancer, infection, or hemoglobinopathy.

\section{Study enrolment and randomization Identifications and invitation}

Participants were identified either when presenting for routine visits or through searching the hospital electronic database for potential patients by using the inclusion/exclusion criteria. After confirming eligibility, the investigator approached eligible participants to ascertain interest. A verbal explanation of the trial and an invitation letter with a copy of the patient information sheet were mailed to interested individuals. This included information about the rationale, design, and personal implications of the study. After information was provided, participants had at least $24 \mathrm{~h}$ to consider whether to participate and were given the opportunity to discuss the trial with their family and health-care professionals before they were invited to attend the screening visit.

\section{Consent and screening}

At the screening visit, eligibility was assessed and written informed consent was obtained by a medically qualified investigator who explained the questionnaire, the procedures required, risks/benefits, and confidentiality to the potential participant prior to obtaining informed consent from willing participants.

Relevant details of participants' medical history (including primary renal diagnosis, iron deficiency status, and concurrent medications) were recorded. Oral iron was discontinued prior to randomization, and participants were instructed to notify the study site about any new medications they took after the start of the study.

After a physical examination, blood and urine samples from willing participants were sent to the hospital's

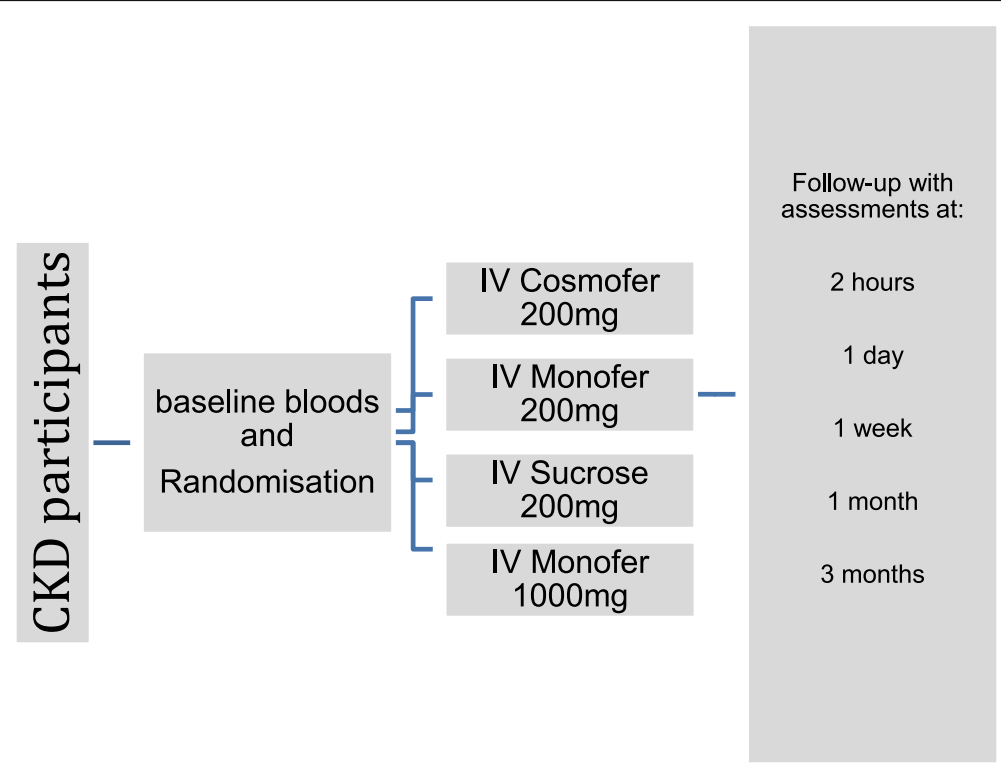

Fig. 1 Flow chart of patient's journey in the trial. Follow-up visits are $2 \mathrm{~h}, 1$ day, 1 week, and 1 month and 3 months after iron infusion. 
Table 1 Inclusion and exclusion criteria

\begin{tabular}{ll}
\hline Inclusion criteria & Exclusion criteria \\
\hline$\geq 18$ years of age & Age $<18$ years \\
$\begin{array}{l}\text { Chronic kidney disease }(\mathrm{CKD}) \text { stages } 3-5 \\
\left.\text { (glomerular filtration rate }<60 \mathrm{~mL} / \mathrm{min} \text { per } 1.73 \mathrm{~m}^{2}\right)\end{array}$ & No renal failure or CKD stages 1-2 \\
$\begin{array}{l}\text { Written and signed informed patient consent and } \\
\text { ability to co-operate with study protocol }\end{array}$ & Patients unable or do not wish to give consent or inability to co-operate with study protocol \\
No previous iron administration in last 6 weeks & Parenteral iron therapy within previous 6 weeks \\
Serum ferritin level less than $200 \mu \mathrm{g} / \mathrm{L}$ & Ferritin greater than $200 \mu \mathrm{g} / \mathrm{L}$ \\
Transferrin saturation $<20 \%$ & Transferrin saturation $>40 \%$ \\
Non-smoker or ex-smoker & Current smokers \\
& Hemochromatosis \\
& $\begin{array}{l}\text { Patients with potential confounding factors to neutrophil gelatinase-associated lipocalin (NGAL) } \\
\text { measurement (cancer, infection) }\end{array}$ \\
& Pregnancy \\
& Patients being investigated for potential blood loss \\
& Patients with a hematological malignancy/ hemolysis or known hemaglobinopathy, including myeloma \\
& Known allergy to any iron therapy \\
\hline
\end{tabular}

pathology laboratory for confirmation of eligibility. If the results were considered inaccurate (e.g., hemolyzed sample) by the investigator, the samples could be repeated once; but if the results did not confirm eligibility, the participant was withdrawn from the study.

\section{Randomization procedure}

After informed consent was obtained, eligible participants were assigned a unique participant identification number. Once this number was assigned to participants, it was not reused. Participants were randomly allocated in a 1:1:1:1 ratio to receive one of the three iron preparations (and two dosing concentrations for Monofer) at visit 2 . The randomization was performed by a computer program in blocks, and the block size was not revealed. Labels were consecutively numbered 1-40. Then they were sealed in non-transparent double-sealed envelopes. Access to these envelopes was not available to investigators. Details of the iron therapy were held by pharmacy that matched the choice of interventional iron therapy with the relevant randomization number. Both numbers and iron therapy treatment administered at visit 2 were recorded in the participants' medical case records.

\section{Baseline measurements}

After randomization, willing and eligible participants were invited to attend a baseline visit, at which baseline investigations were performed (Table 2). These included PWV, eGFR, biochemical profile (BCP), full blood count (FBC), SF, TS\%, and C-reactive protein (CRP). Two further samples of plasma and serum were obtained from participants and stored at $-80{ }^{\circ} \mathrm{C}$ for future analysis of NGAL, oxidative stress, and inflammatory markers at the University of Hull. Samples were kept on ice in a sealed container when transported from the hospital outpatient clinic to the hospital's pathology laboratory or from the hospital to the university.

At baseline, participants were asked to complete a preference-based measure of health (SF-36) questionnaire, a standardized survey used to assess patient health across eight dimensions [20]. After all baseline measurements were performed, participants were accompanied to the hospital's medical day unit, where IV iron was administered in accordance with hospital policy.

\section{Iron administration}

Participants were randomly assigned to receive one of the four options. Infusions consisting of $200 \mathrm{mg}$ of the iron preparation Venofer, Cosmofer, or Monofer or an infusion of $1000 \mathrm{mg}$ of Monofer were supplied by hospital pharmacy in accordance with departmental protocol. All preparations were administered over a 1-h time period apart from the low-dose Monofer, which was given over $15-20 \mathrm{~min}$ in accordance with normal practice at Hull and East Yorkshire Hospitals NHS Trust (renamed Hull University Teaching Hopsitals NHS Trust). Patients were closely monitored throughout the infusion and $30 \mathrm{~min}$ later for hemodynamic changes or side effects. Two hours after the infusion, participants were reviewed again and all investigations performed at baseline (except the health questionnaire) were repeated. Participants were monitored closely for adverse effects throughout the study and any events were recorded in the electronic case report and in the medical notes. 
Table 2 Summary of schedule detailing all of the assessments required at each visit

\begin{tabular}{|c|c|c|c|c|c|c|c|}
\hline \multirow[t]{2}{*}{ Procedures } & Visit 1 & Visit 2a & Visit 2b & Visit 3 & Visit 4 & Visit 5 & Visit 6 \\
\hline & Screen & baseline & Post iron $2 \mathrm{~h}$ & 1 day & 1 week & 1 month & 3 month \\
\hline Informed consent & $x$ & & & & & & \\
\hline Demographics & $x$ & & & & & & \\
\hline History & $x$ & & & & & & \\
\hline Examination & $x$ & & & & $x$ & $x$ & $x$ \\
\hline Weight & $x$ & $x$ & & $x$ & $x$ & $x$ & $x$ \\
\hline $\mathrm{BCP}$ & $x$ & $x$ & $x$ & $x$ & $x$ & $x$ & $x$ \\
\hline Albumin & $x$ & $x$ & $x$ & $x$ & $x$ & $x$ & $x$ \\
\hline eGFR & $x$ & $x$ & $x$ & $x$ & $x$ & $x$ & $x$ \\
\hline FBC & $x$ & $x$ & $x$ & $x$ & $x$ & $x$ & $x$ \\
\hline Ferritin & $x$ & $x$ & $x$ & $x$ & $x$ & $x$ & $x$ \\
\hline TS\% & $x$ & $x$ & $x$ & $x$ & $x$ & $x$ & $x$ \\
\hline CRP & $x$ & $x$ & $x$ & $x$ & $x$ & $x$ & $x$ \\
\hline Cystatin C & & $x$ & $x$ & $x$ & $x$ & $x$ & $x$ \\
\hline UACR/UPCR & & $x$ & $x$ & $x$ & $x$ & $x$ & $x$ \\
\hline NGAL & & $x$ & $x$ & $x$ & $x$ & $x$ & $x$ \\
\hline Oxidative stress markers & & $x$ & $x$ & $x$ & $x$ & $x$ & $x$ \\
\hline Pulse wave velocity & & $x$ & $x$ & $x$ & $x$ & $x$ & $x$ \\
\hline SF-36 & & $x$ & & & $x$ & $x$ & $x$ \\
\hline Medication check & & $x$ & & & $x$ & $x$ & $x$ \\
\hline Adverse events & & & $x$ & $x$ & $x$ & $x$ & $x$ \\
\hline
\end{tabular}

" $\mathrm{X}$ " indicates action to be taken or an investigation to be performed

Abbreviations: $B C P$ biochemical profile (consisting of urea and electrolytes, creatinine, liver function tests, albumin, total protein, calcium, phosphate, and bicarbonate), CRP C-reactive protein, eGFR estimated glomerular filtration rate, FBC full blood count (consisting of hemoglobin, white cell count, platelets, mean cell volume, and mean cell hemoglobin concentration), NGAL neutrophil gelatinase-associated lipocalin, SF-36 36-Item Short Form Health Survey, TS\% transferrin saturation percentage, $u A C R / U P C R$ urinary albumin:creatinine ratio/urinary protein:creatinine ratio

\section{Assessments}

At all visits, the investigator sought the information on adverse events (serious and non-serious) considered by participants to be related to iron therapy. In addition to documenting changes in health and concurrent medications, two participants who were considered to require further iron infusions by the caring nephrologist whilst still on the study were withdrawn. Weight, blood pressure, temperature, and PWV were measured at all visits, and the SF-36 questionnaire was completed at 1-month and 3-month visits (Table 2).

Blood levels to measure $\mathrm{Hb}$, iron markers, CRP, and kidney function were performed, as in normal practice. Quantification of proteinuria was carried out by measurement of urinary protein:creatinine ratio (uPCR) or, if diabetic, urinary albumin:creatinine ratio (uACR) levels in a spot urine sample by using standard laboratory techniques.

Additional samples from EDTA and serum-separating tubes were centrifuged and the plasma and serum aliquoted into cryovials, which were initially stored locally at $-80{ }^{\circ} \mathrm{C}$ prior to transfer to the university laboratory in Hull, UK, where they will be stored at $-80{ }^{\circ} \mathrm{C}$ until analysis for inflammatory and oxidative stress biomarkers.
After 3 months of study follow-up, participants will continue to be reviewed in accordance with normal practice three times a month. All information was collected and recorded on a secure encrypted and password-protected computer database in the research unit at the NHS Trust.

\section{Enzyme-linked immunosorbent assay for quantitative detection of NGAL}

NGAL will be measured by enzyme-linked immunosorbent assays (Life Technologies | Thermo Fisher Scientific, Carlsbad, CA, USA) for quantitative detection of human NGAL and expressed as nanograms per milligram per creatinine. In brief, the plates are precoated with primary NGAL antibody and therefore ready to use. The calibrators and dilated sample are added to the wells and incubated for $30 \mathrm{~min}$. Tetramethylbenzidine or a substitute is added to each well along with a stop solution after $15 \mathrm{~min}$ and then quantum results are obtained by measuring the absorbance at $450 \mathrm{~nm}$.

\section{Oxidative stress biomarkers}

Plasma malondialdehyde (MDA) levels will be measured by derivatization with thiobarbituric acid by using an isocratic high-performance liquid chromatography technique. 


\section{F2-isoprostanes}

A competitive immunoassay with colorimetric quantification (Direct 8-iso-Prostaglandin F2 $\alpha$ Enzyme Immunoassay Kit, Assay Designs, Enzo Life Sciences, Farmingdale, NY, USA) will be used to measure 8-iso-prostaglandin F2 $\alpha$ (8-iso-PGF2 $\alpha$ ), also termed F2-isoprostane, as a marker of lipid peroxidation.

\section{Non-transferrin-bound iron and labile plasma iron}

Levels of non-transferrin-bound iron (NTBI) and labile plasma iron (LPI) will be measured by using the FeROS ${ }^{\text {тм }}$ assay (Aferrix Ltd., Tel-Aviv, Israel)..This assay employs a selective iron chelator that blocks redox cycling of iron to specifically identify iron-mediated reactive oxygen species generation. Comparison of the fluorescence generated in the reaction in the presence and absence of the iron chelator translates into an accurate estimate of the quantity of LPI in the tested sample.

\section{Monitoring}

Prior to recruitment, study staff received training in the study procedures. The study has been monitored in accordance with Hull and East Yorkshire Research and Development Department standard operating procedures to ensure compliance with UK Clinical Trial Regulations. Deviations from the protocol or good clinical practice were reported by the investigator to the Department (as sponsor) on monitoring report forms every two months. Investigators will take into account all protocol deviations and any serious breaches in the final study analysis and publication.

\section{Statistical considerations}

\section{Sample size calculation}

As this was an explorative pilot study looking for proof of concept, a size calculation was not required. There are no previous studies examining the differential impact of different intravenous iron preparations on markers of AKI. Other studies examining AKI using NGAL, have demonstrated that a plasma NGAL (at a cutoff value of $50 \mu \mathrm{g} / \mathrm{L}$ ) were powerful independent predictors of AKI, with an area under the receiver operating characteristic curve of 0.91 [16]. Therefore, one would anticipate a large change from baseline of NGAL values as a result of intravenous iron if the hypothesis is correct.

\section{Statistical analysis}

All analyses will involve comparing outcomes and changes in the different parameters during the scheduled study visits among all participants randomly assigned to receive one of the four iron therapies. Comparisons of continuous outcomes (including the primary outcome) between the allocated treatment arms will be performed by using analysis of covariance (ANCOVA) adjusted for each patient's value at baseline. Multiple imputation techniques with such a small study will be of limited value. Therefore, we will analyze all available data without data replacement as these are missing at random rather than related to the trial intervention. The safety population will include any randomly assigned participants who received any amount of study drug. Descriptive statistics and graphic approach will be employed for the exploratory analysis of variations in oxidative stress, inflammatory markers, and markers of acute kidney injury. In addition, we will explore the impact of diabetes and gender, but because of the size of this explorative study, we will examine the impact of diabetes $(n=13)$ in the whole cohort versus non-diabetes $(n=27)$ but this analysis will be simply hypothesis-generating rather than conclusive. Being on lipid-lowering medication such as statins may affect lipid peroxidation and activities of antioxidant enzymes. Broncel et al. [21] have shown that in patients with dyslipidemia without clinical symptoms of atherosclerosis, atorvastatin, simvastatin, and pravastatin decreased similar thiobarbituric acid-reactive substance (TBARS) concentrations in the isolated erythrocyte membranes. The authors observed a significant increase of the antioxidant enzyme activities during atorvastatin and simvastatin treatment. Therefore, this will be examined to see whether there is an impact in those patients but again numbers will be small.

\section{Results}

Between October 2015 and April 2018, 521 individuals were identified as potential patients by using lists of patients awaiting IV iron therapy and using an electronic database of patients with CKD and long-term iron deficiency; 237 (45.5\%) were found to live locally. Of these, 216 (91\%) were contacted by telephone after receiving an information sheet and 56 (26\%) expressed interest in taking part in the study. Most of these patients lived locally with a maximum of 30 -min driving time to the hospital, which has contributed to their compliance throughout the study. Another major factor that contributed to patient compliance was their mobility and access to transport (i.e., regardless their age or gender). We found that the more independently mobile the patients were and the easier their access to private or public transport was, the more likely they were to consent to take part in a study with six visits over a 3-month period. Forty-nine patients attended a screening visit, which usually coincided with their usual follow-up nephrology appointment. Seven of them did not meet the eligibility criteria following screening and this was due mainly to iron markers being normal or above the required range. Two patients felt that the study contained too many visits and therefore decided not to continue taking part. Eventually, 40 were confirmed to meet the eligibility criteria (Fig. 2). All eligible patients were randomly assigned to intervention with one of the four iron 


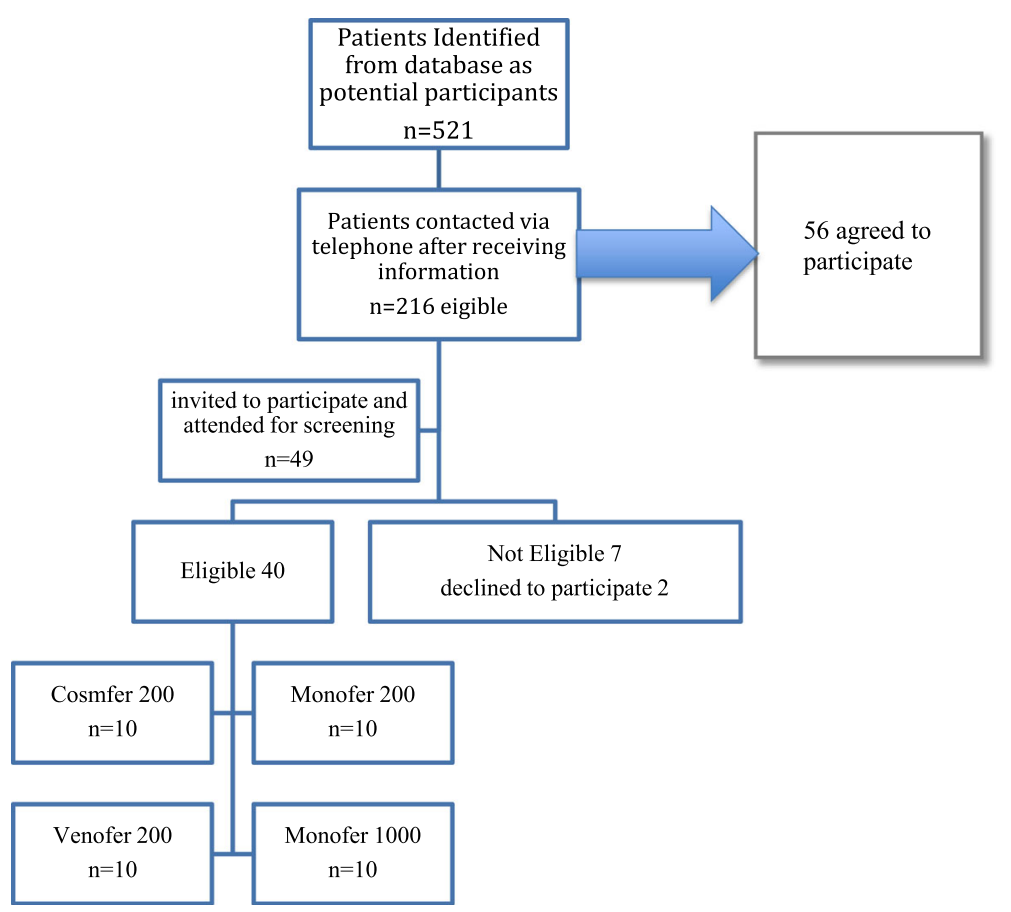

Fig. 2 Consort figure of flow of patients through IRON-CKD trial

preparations and then followed up at $2 \mathrm{~h}, 1$ day, 1 week, 1 month, and 3 months after the infusion.

\section{Baseline characteristics of randomly assigned participants} In total, 40 patients have been randomly assigned. The mean age was 58.3 years (standard error of the mean (SEM) 4.4.) and 23 (58\%) were male (Table 3). The mean SF was $66.6 \mu \mathrm{g} / \mathrm{L}$ (SEM 15), TS 21.2\% (SEM 3.2), and $\mathrm{Hb} 121.6 \mathrm{~g} / \mathrm{L}$ (SEM 5.46) at randomization for the whole group. The mean eGFR was $27.8 \mathrm{~mL} / \mathrm{min}$ per $1.73 \mathrm{~m}^{2}$ (SEM 4), uACR $110.4 \mathrm{mg} / \mathrm{mmol}$ (SEM 65.3), and uPCR
$104.3 \mathrm{mg} / \mathrm{mmol}$ (SEM 67). CRP was $6.7 \mathrm{mg} / \mathrm{L}$ (SEM 2.3). The etiology of the renal failure varied between the patients and is detailed in Table 4.

\section{Discussion}

The relative safety of IV iron preparations regarding acute infusion-related effects is not well characterized. A few retrospective epidemiological studies have explored the relative risks of serious adverse events associated with IV iron products. A main knowledge gap in the literature is the lack of head-to-head randomized controlled studies

Table 3 Baseline demographic data

\begin{tabular}{|c|c|c|c|c|c|}
\hline & Venofer 200 & Cosmofer 200 & Monofer 200 & Monfer 1000 & Total group \\
\hline Age, years & $49.5(4.3)$ & $66.2(2.3)$ & $59.6(5.0)$ & $58.8(5.9)$ & $58.3(4.4)$ \\
\hline Male/female, numbers & $6 / 4$ & $6 / 4$ & $4 / 6$ & $7 / 3$ & $23 / 17$ \\
\hline Serum ferritin, $\mu \mathrm{g} / \mathrm{L}$ & $58.5(11.6)$ & $80.8(20.6)$ & $60.5(9.5)$ & $66.6(18.4)$ & $66.6(15.0)$ \\
\hline Transferrin saturation percentage & $21.1(2.9)$ & $25.4(4.9)$ & $21.2(3.1)$ & $18.1(1.7)$ & $21.2(3.2)$ \\
\hline Hemoglobin & $121.4(4.8)$ & $129.1(7.4)$ & $122.5(5.0)$ & $115.4(4.7)$ & $121.6(5.5)$ \\
\hline Serum creatinine, micromole/L & $278.7(42.8)$ & $255.8(39.6)$ & $175.6(16.8)$ & $258.5(30.5)$ & $242.2(51.2)$ \\
\hline eGFR, mL/min per $1.73 \mathrm{~m}^{2}$ & $25.7(4.8)$ & $26.7(3.9)$ & $32.9(3.8)$ & $25.9(3.6)$ & $27.8(4.0)$ \\
\hline $\mathrm{uACR}, \mathrm{mg} / \mathrm{mmol}$ & $45.8(24.5)$ & $11.2(5.76)$ & $234.0(154)$ & $145.6(77.1)$ & $110.1(65.3)$ \\
\hline $\mathrm{uPCR}, \mathrm{mg} / \mathrm{mmol}$ & $236.3(104.5)$ & $117.5(68.1)$ & $20(0.5)$ & $164(94.6)$ & $104.3(67)$ \\
\hline C-reactive protein, mg/L & $8.8(3.3)$ & $5.6(1.9)$ & $7.7(2.9)$ & $4.6(1.0)$ & $6.7(2.3)$ \\
\hline Albumin, $g / L$ & $34.6(3.7)$ & $37.1(3.2)$ & $35.2(3.8)$ & $32.6(3.5)$ & $34.88(3.55)$ \\
\hline
\end{tabular}

Mean values and the standard error of the mean for each of the four intravenous iron groups ( $n=10$ per group) 
Table 4 Etiology of renal disease in whole cohort of patients

\begin{tabular}{llllll}
\hline & Cosmofer 200 & Venofer 200 & Monofer 200 & Monofer 1000 \\
\hline Diabetes & 2 & 4 & 4 & 3 & 13 \\
Hypertension & 2 & 1 & 2 & 5 & 10 \\
Polycystic kidney disease & 0 & 2 & 2 & 1 & 5 \\
Pyelonephritis & 0 & 1 & 0 & 0 & 2 \\
Glomerulonephritis & 2 & 0 & 2 & 0 & 6 \\
Other & 4 & 2 & 0 & 6
\end{tabular}

comparing IV preparations; thus, true comparative analysis is difficult. Therefore, further studies are needed. This small randomized open-label study will provide valuable information for clinicians to consider.

\section{Conclusions}

The IRON-CKD study will provide further important clinical and experimental data to allow some evaluation of the relative acute effects associated with Venofer, Cosmofer, and Monofer (low- and high-dose) on markers of oxidative stress, inflammation and acute kidney injury and potentially differences in the iron preparations acknowledging the small numbers. The data will be hypothesis-generating. In addition, data will be generated on the relative efficacy of these agents for improving $\mathrm{Hb}$ concentrations and again this may suggest simply a possible difference. Finally, the study will examine measures of endothelial function and quality of life among patients with non-dialysis-dependent CKD. Recent publications have shown variations in effects and this study will add further clinical and mechanistic data to allow one to differentiate the iron products. The future use of the data from this study may allow one to power/design a larger study which will permit investigators to compare these iron preparations in a more statistically and clinically meaningful fashion.

\section{Abbreviations}

AKI: Acute kidney injury; CKD: Chronic kidney disease; CRP: C-reactive protein; EDTA: Ethylenediaminetetraacetic acid; eGFR: Estimated glomerular filtration rate; Hb: Hemoglobin; IV: Intravenous; LPI: Labile plasma iron; NGAL: Neutrophil gelatinase-associated lipocalin; NHS: National Health Service; PWV: Pulse wave velocity; RES: Reticulo-endothelial system; SEM: Standard error of the mean; SF: Serum ferritin; SF-36: 36-Item Short Form Health Survey; TS: Transferrin saturation; UACR: Urinary albumin:creatinine ratio; UPCR: Urinary protein:creatinine ratio

\section{Acknowledgments}

We would like to thank Tracy Cathcart and the research team for their assistance during the study.

\section{Funding}

The IRON-CKD study received an unrestricted grant from Pharmacosmos. The funders had no role in study design, data collection and analysis, decision to publish, or preparation of the manuscript.

\section{Availability of data and materials}

All data that are anonymized will be available from AZ with the relevant permissions and agreement of the Research and Development Committee of Hull and East Yorkshire Hospitals NHS Trust.

\section{Authors' contributions}

AZ contributed to this manuscript and approval of this submission and wrote the first draft of the study protocol. SB participated in all aspects of the study, obtained funding for the study, and critically revised the manuscript. The order of authorship has been a joint decision of the co-authors based on substantial contribution to conception and design, execution, analysis, and interpretation of data. SB is the senior author. Both authors read and approved the final manuscript.

\section{Authors' information}

Not applicable.

\section{Ethics approval and consent to participate}

The study was given a favorable opinion from the Northern Regional Ethics Service (NRES) Committee Yorkshire and the Humber - Leeds East, UK (approval reference number 10/H1306/40). Study participants have all details explained to them in writing and in person before giving informed consent.

\section{Consent for publication}

Neither this article nor substantial parts of it are under consideration for publication elsewhere or have been published or made available elsewhere in a manner that could be construed as a prior or duplicate publication of the same content.

\section{Competing interests}

AZ declares that he has no competing interests. SB has received honorarium for lectures, attended expert opinion committees, and received educational funds to attend the European Society of Dialysis and Transplantation (EDTA annual scienctic meeting 2016 and 2017) from Pharmacosmos and Vifor Pharma.

\section{Publisher's Note}

Springer Nature remains neutral with regard to jurisdictional claims in published maps and institutional affiliations.

Received: 21 August 2018 Accepted: 14 March 2019

Published online: 04 April 2019

\section{References}

1. Cox HJ, Bhandari S, Rigby AS, Kilpatrick ES. Mortality at low and high estimated GFR values. A U- shaped curve. Nephron Clin Pract. 2008;110:67-72.

2. Sarnak MJ. Clinical epidemiology of cardiovascular disease in chronic renal disease. Am J Kidney Dis. 1998;32:S 112-9.

3. Bhandari S, Norfolk D, Brownjohn A, Turney J. Evaluation of red blood cell ferritin and reticulocyte measurements in monitoring response to intravenous iron therapy. Am J Kidney Dis. 1997;30(6):814-21.

4. Besarab A, Amin N, Ashan M, Vogel SE, Zazuwa, Frinak S, et al. Optimisation of epoetin therapy with intravenous iron supplementation in haemodialysis patients. J Am Soc Nephrol. 2000;11:530-8.

5. Bhandari S, Naudeer S. Improving efficiency and value in Health Care. Intravenous iron management for anaemia associated with chronic kidney disease. Linking treatment to an outpatient clinic, optimising service provision and patient choice. J Eval Clin Pract. 2008;14:996-1001.

6. Shah SV, Baliga R, Fonseca VA, Rajapurkar M. Oxidants in chronic kidney disease. J Am Soc Nephrol. 2007;18:16-28.

7. Halliwell B, Gutteridge JMC. Role of free radicals and catalytic metal ions in human disease: an overview. Meth Enzymol. 1990;186:1-85. 
8. Rajapurkar MM, Baliga R, Shah SV. Treatment of patients with glomerulonephritis with an oral iron chelator [abstract]. J Am Soc Nephrol. 2007;18:57-58A.

9. Lin J-L, Lin-Tan D-T, Hsu K-H, Yu C-C. Environmental lead exposure and progression of chronic renal diseases in patients without diabetes. N Engl J Med. 2003;348:277-86.

10. Silverstein SB, Rodgers GM. Parenteral iron preparations. Am J Haematol. 2004:76:74-8.

11. Zager RA, Johnson AC, Hanson SY, Wasse H. Parenteral iron formulations: a comparative toxicological analysis and mechanisms of cell injury. Am J Kid Dis. 2002:40:90-103.

12. Zager RA, Johnson AC, Hanson SY. Parenteral iron nephrotoxicity, potential mechanisms and consequences. Kidney Int. 2004;66:144-56.

13. Jahn MR, Andreasen HB, Fütterer S, Nawroth T, Schünemann V, Kolb U, et al. A comparative study of the physicochemical properties of iron isomaltoside 1000 (Monofer), a new intravenous iron preparation and its clinical implications. Eur J Pharm Biopharm. 2011;78:480-91.

14. Mori K, Nakao K. Neutrophil gelatinase-associated lipocalin as the real-time indicator of active kidney damage. Kidney Int. 2007;71:967-70.

15. Kjeldsen $L$, Johnsen $A H$, Sengelov $H$, Borregaard N. Isolation and primary structure of NGAL, a novel protein associated with human neutrophil gelatinase. J Biol Chem. 1993:268:10425-32.

16. Mishra J, Dent C, Tarabishi R, Mitsnefes MM, Ma Q, Kelly C, et al. NGAL as a biomarker of acute renal injury after cardiac surgery. Lancet. 2005;65:1231-8.

17. Bachorzewska-Gajewska H, Malyszko J, Sitniewska E, Malyszko JS, Dobrzyckin S. Neutrophil gelatinase-associated lipocalin (NGAL) correlations with cystatin $C$ serum creatinine and eGFR in patients with normal serum creatinine undergoing coronary angiography. Nephrol Dial Transplant. 2007;22:295-6.

18. Mishra J, Mori K, Ma Q, Kelly C, Barasch J, Devarajan P. Neutrophil gelatinase-associated lipocalin: a novel early urinary biomarker for cisplatin nephrotoxicity. Am J Nephrol. 2004;24:307-15.

19. Mikhail A, Brown C, Williams JA, Mathrani V, Shrivastava R, Evans J, et al. Renal association clinical practice guideline on Anaemia of Chronic Kidney Disease. BMC Nephrol. 2017;18:345.

20. Gandek B, Sinclair SJ, Kosinski M, Ware JE. Psychometric Evaluation of the SF $36^{\circ}$ Health Survey in Medicare Managed Care. Health Care Financ Rev. 2004;25:5-25.

21. Broncel M, Koter-Michalak M, Chojnowska-Jezierska J. The effect of statins on lipids peroxidation and activities of antioxidants enzymes in patients with dyslipidemia. Przeglad Lekarski. 2006;63:738-42 Retrieved from http:// www.ncbi.nlm.nih.gov/pubmed/17479860

Ready to submit your research? Choose BMC and benefit from:

- fast, convenient online submission

- thorough peer review by experienced researchers in your field

- rapid publication on acceptance

- support for research data, including large and complex data types

- gold Open Access which fosters wider collaboration and increased citations

- maximum visibility for your research: over $100 \mathrm{M}$ website views per year

At $\mathrm{BMC}$, research is always in progress.

Learn more biomedcentral.com/submissions 